\title{
Stress relief behaviour of diamond-like carbon films on glasses
}

\author{
Kwang-Ryeol Lee, Young-Joon Baik and Kwang-Yong Eun \\ Ceramics Processing Laboratory, Korea Institute of Science and Technology, PO Box 131, Cheongryang, Seoul (South Korea)
}

\begin{abstract}
The stress relief behaviour of diamond-like carbon (DLC) films on glass substrates was investigated. The r.f.-plasma-enhanced chemical vapour deposition method was used for the deposition with variable negative bias voltages of the cathode from -100 to $-700 \mathrm{~V}$. Beyond a critical thickness of the film, film delamination starts to occur after its removal from the reaction chamber. In addition to the previously observed stress relief morphologies, sinusoidal cracking and sinusoidal removal of the film are also observed. The stress relief morphology is dependent on the film thickness and the negative bias voltage. The measured wavelength of sinusoidal buckling increases with both increasing film thickness and increasing negative bias voltage. This behaviour is qualitatively consistent with a phenomenological equation derived by Nir. It was suggested that the elastic modulus of the DLC film can be calculated using the dependence of the wavelength of sinusoidal buckling on the film thickness and the negative bias voltage.
\end{abstract}

\section{Introduction}

Since the first report of Aisenberg and Chabot [1], diamond-like carbon (DLC) films have attracted considerable interests owing to their unique combination of properties. Among the important properties of these films are high hardness, low coefficient of friction, chemical inertness, optical transparency and high electrical resistivity. The unusual combination of these properties has stimulated studies on various applications such as optical coatings, high endurance, low friction coatings and diffusion barriers for electronic components [2-5]. However, it is well known that typical films have high intrinsic compressive stresses of up to $10 \mathrm{GPa}$, which result in the delamination of thick films from their substrates [6]. Therefore the large compressive stress has been one of the obstacles to the application of DLC coatings.

The stress relief behaviour of DLC films has been reported to have the characteristic morphology of sinusoidal buckling propagation. In 1979 Weissmantel and co-workers $[7,8]$ first reported sinusoidal stress relief patterns of DLC films on glass and $\mathrm{NaCl}$ substrates. Their study was, however, limited to the observation itself. Later, Nir [9] investigated the shapes of stress relief patterns such as sinusoidal buckling, straight cracking, strings of beads and cracking after buckling with theoretical analysis based on the linear theory of thin plates. Nir reported the qualitative dependence of the dimension of the buckling patterns on the film thickness. However, the effects of the film thickness and film properties on the stress relief morphology were not fully investigated. Although additional observations were made on the stress relief behaviour of DLC films [10], thorough investigations based on in-depth observations have not yet been reported.

We have studied the stress relief behaviour of DLC films on glass substrates. The object of this study was to investigate a relationship between the stress relief morphology and the process parameters. The negative bias voltage on the cathode is one of the most important process parameters in r.f.-plasma-enhanced chemical vapour deposition (r.f.-PECVD) that determines the ion energy and thus the film structure and properties [11]. Hence the stress relief behaviour of DLC films was investigated in terms of the variation in the negative bias voltage. The changes in stress relief morphology with increasing film thickness are also illustrated in this paper.

Delamination of compressively stressed films by buckling or cracking is a common phenomenon of many metallic and ceramic films [12]. Recently, sinusoidal stress relief patterns were also reported in a model experiment of a mica film on $\mathrm{Al}$ [13]. In the case of a DLC film deposited on a glass substrate, both the film and the substrate are elastically isotropic amorphous phases, which offers the simplest case for a theoretical treatment. Hence investigations on the buckling behaviour of DLC films on glass substrates will help us to extend our understanding of this general phenomenon.

\section{Experimental methods}

Diamond-like carbon films were deposited in r.f. glow discharges of $\mathrm{CH}_{4}$ gas. A schematic diagram of the 
experimental set-up is shown in Fig. 1. The electrode is water-cooled stainless steel, $12.5 \mathrm{~cm}$ in diameter, on which the substrates to be coated are placed. The discharge is sustained by $13.56 \mathrm{MHz}$ r.f. power supplied to the electrode via a matching network. In order to confine the glow discharge, the electrode is surrounded by a ground shield which is extended to the gas supply ring.

A precleaned slide glass (Corning 2948) of size $25 \mathrm{~mm} \times 75 \mathrm{~mm}$ was used as the substrate. In order to measure the film thickness, part of the slide glass was masked by a cover glass. The film thickness, which is the height of the step formed by the mask, was measured by an Alphastep profilometer. The chamber was evacuated to $10^{-5}$ Torr by a turbomolecular pump. Before deposition the substrate was cleaned in situ by an $\mathrm{Ar}$ discharge at $-400 \mathrm{~V}$ and $3 \times 10^{-3}$ Torr for $15 \mathrm{~min}$. Methane was then supplied at a fixed flow rate of $8 \mathrm{sccm}$ (standard $\mathrm{cm}^{3} \mathrm{~min}^{-1}$ ). The deposition pressure, measured by a capacitance manometer, was $9 \times 10^{-3}$ Torr.

The negative bias voltage was varied from -100 to $-700 \mathrm{~V}$ by adjusting the r.f. power supply. Depending on the negative self-bias voltage, deposition was performed for 5-180 min. The stress relief behaviour was observed by an optical microscope. Because buckling propagation occurs over a time interval of the order of minutes, in situ observation under the optical microscope was possible. However, the microstructures shown in this paper were taken about 1 month after the deposition and thus show the stabilized morphology.

In order to measure the stress of the film, thin $\mathrm{Si}(100)$ wafers about $200 \mu \mathrm{m}$ thick were used as the substrates. The stress was calculated from the equilibrium equation

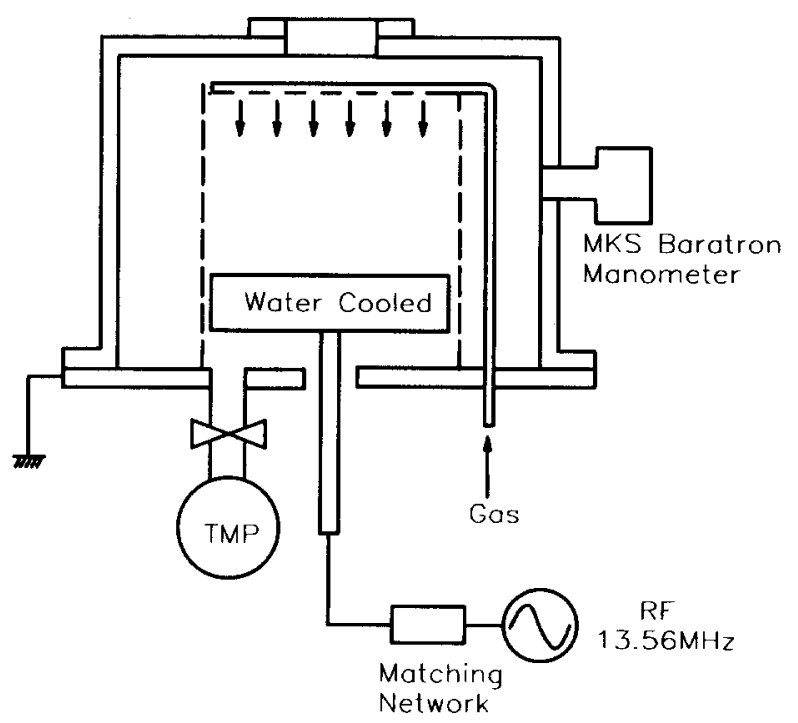

Fig. 1. Schematic diagram of the r.f.-PECVD apparatus used in this work. of bending plates [14]:

$\sigma=\frac{E D^{2}}{6 r h(1-v)}$

where $\sigma$ is the stress in the film, $D, E$ and $v$ are the thickness, elastic modulus and Poisson ratio of the substrate respectively and $r$ and $h$ are the radius of curvature of the film-substrate composite and the thickness of the film respectively. The curvature of the filmsubstrate composite was measured by the laser reflection method. A value of $1.8 \times 10^{2} \mathrm{GPa}$ was used for $E /(1-v)$ of the Si substrate. The thickness of the substrate was measured by scanning electron microscopy (SEM). Films with a minimum thickness of $1.5 \mu \mathrm{m}$ were used for the measurements. The stresses in the deposited films were independent of the thickness. The error in the stress measurement is estimated to be at most $10 \%$.

\section{Results and discussion}

The deposited films display high hardness, transparency and low friction coefficient typical of DLC films. The colours of the films range from pale yellow at low negative bias voltage to dark brown at high negative bias voltage. The total hydrogen content of the film was measured by combustion element analysis of carbon, hydrogen and nitrogen [15]. The hydrogen content ranges from 32 at. $\%$ at $-700 \mathrm{~V}$ to 42 at. $\%$ at $-100 \mathrm{~V}$. For thin films where delamination does not occur at all or occurs only slowly, the thicknesses of the films can be determined by measuring the step heights. The film thickness is linearly proportional to time in these films, showing that steady state deposition is achieved. The absence of a colour fringe in the film and the film thickness measurement show that our film has a uniform thickness. Figure 2 shows the dependence of the deposition rate on the negative self-bias voltage. The rate is linearly proportional to the negative bias voltage within the range from -100 to $-700 \mathrm{~V}$. For thick films where delamination occurs rapidly, thickness determination by measuring the step height was impossible. In these cases the film thicknesses were estimated from the deposition rates and deposition times.

At or beyond a certain critical thickness, delamination of the film starts to occur after its removal from the reaction chamber. As in previous observations [9], buckling starts from the specimen edges or defects and is enhanced by high humidity. Figure 3 shows a typical microstructure of the stress relief patterns: sinusoidal buckling, branching, a string of beads which develops from a straight crack, and sinusoidal cracking. Each mode is indicated by the numbered arrow-heads in Fig. 3 .

The sinusoidal buckling propagates in a random direction; this is a natural consequence of the isotropic 


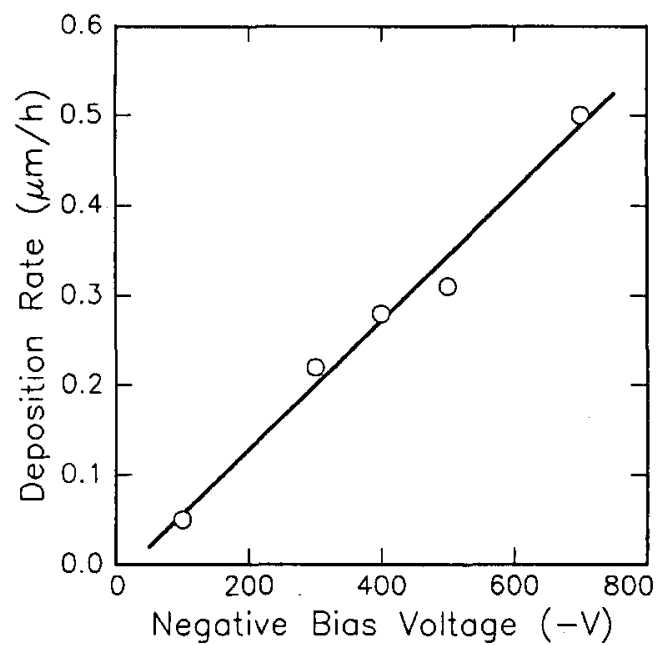

Fig. 2. Dependence of the deposition rate on the negative bias voltage of the cathode.

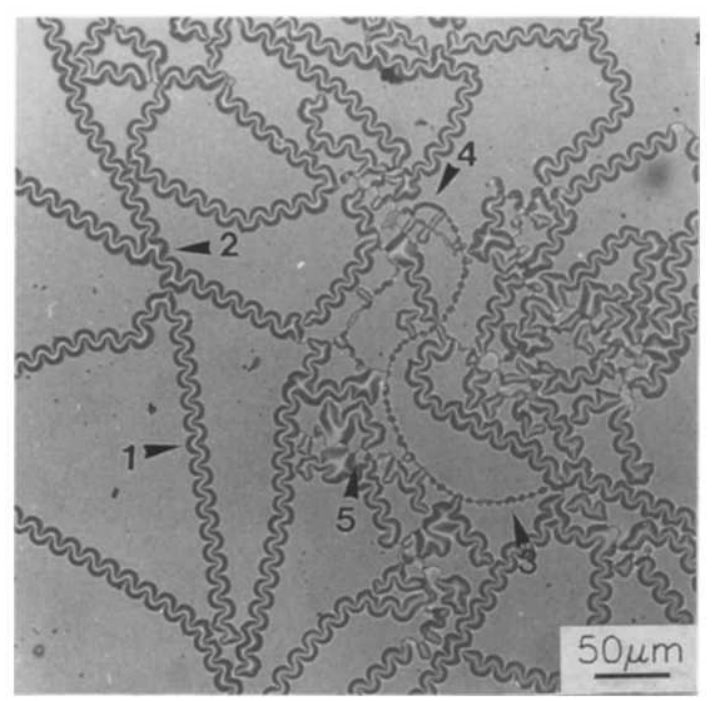

Fig. 3. Microstructure of a typical stress relief morphology in DLC films deposited at a negative bias voltage of $-300 \mathrm{~V}$ for $45 \mathrm{~min}$ : 1, sinusoidal buckling; 2 , branching; 3 , string of beads; 4 , straight cracking; 5 , sinusoidal cracking.

stress in the film. Furthermore, the amorphous substrate cannot be expected to play any role in constraining the propagation direction. The parallel sinusoidal buckling on a glass substrate observed by both Weissmantel and co-workers [7, 8] and Nir [9] cannot be observed in the present work. As for the parallel buckling waves, the presence of anisotropic internal stress might be the reason, as Nir points out [9]. However, the present result shows that the sinusoidal buckling is inherent to the compressively stressed film regardless of the existence of anisotropic internal stresses.

In addition to the straight cracking and string of beads which were previously reported [9], sinusoidal cracking is also frequently observed (arrow-head 5 in Fig. 3). From this crack, additional buckling waves start to propagate. Figure 4 shows a high magnification microstructure of a sinusoidal crack from which buckling waves are propagating. In situ observation shows that the sinusoidal crack occurs along the highest line of the sinusoidal buckling. The wavelength of this crack is thus identical to that of the sinusoidal buckling waves.

As the film thickness increases, the delamination mode changes from mainly sinusoidal buckling at first, then to a mixture of buckling and cracking and finally to mainly cracking. During the initial stage of delamination, however, the sinusoidal buckling is dominant in all cases. For thicker films, cracking occurs as the delamination process takes place. Figure 5 shows the dependence of the delamination morphology on the film thickness for negative bias voltages of -100 and $-500 \mathrm{~V}$. Except for the dimensions of sinusoidal buckling and sinusoidal cracking, their behaviours are identical. Figures 5(c) and 5(d) show that the sinusoidal cracking is mixed with sinusoidal buckling, which is coarsened after it covers the whole area of the specimen. However, the cracking morphologies of thicker films are different from each other as shown in Figs. 5(e) and 5(f). For the $-100 \mathrm{~V}$ sample, sinusoidally buckled regions of the film are removed from the substrate. However, for the film formed at higher negative bias voltage, delamination occurs as random cracks with buckling between them. These random cracks occur along the boundary of sinusoidal buckling as can be seen from in situ observations. Some broken film is also observed to be removed from the substrate.

We have measured the wavelength and amplitude of sinusoidal buckling with respect to the film thickness for various values of negative bias voltage. The ratio of the wavelength to the amplitude is about 4 regardless of the

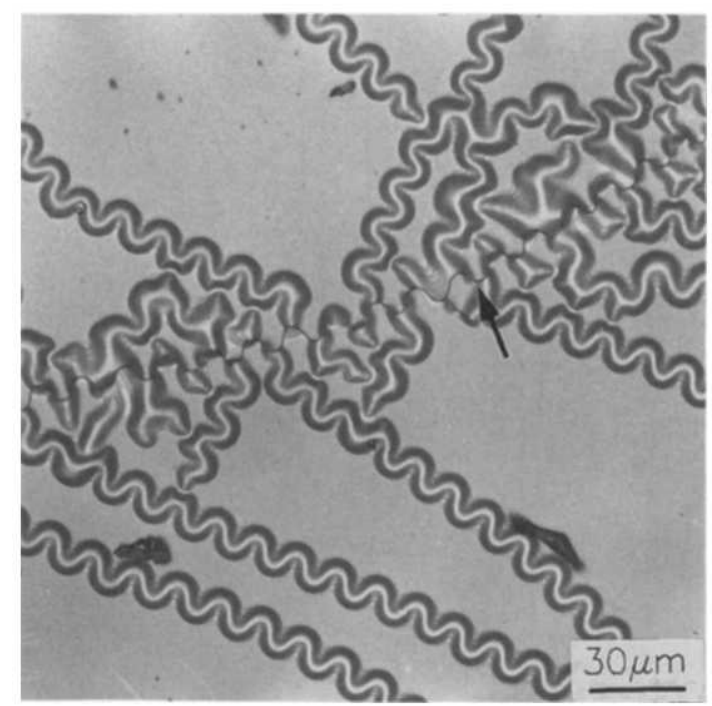

Fig. 4. Microstructure of sinusoidal ctacking with sinusoidal buckling in a DLC film deposited at a negative bias voltage of $-300 \mathrm{~V}$ for $45 \mathrm{~min}$. Sinusoidal cracking is indicated by an arrow. 


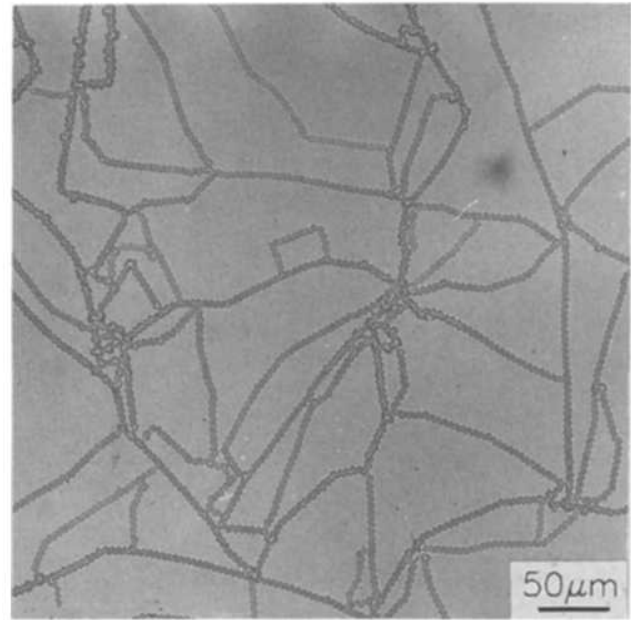

(a)

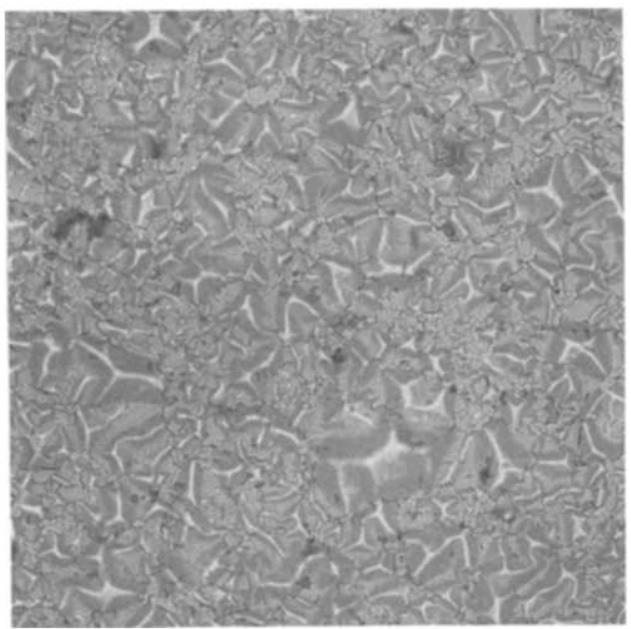

(c)

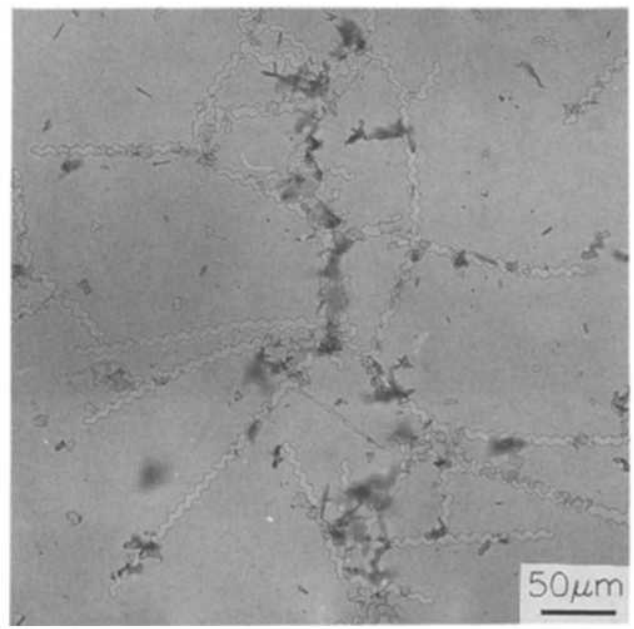

(e)

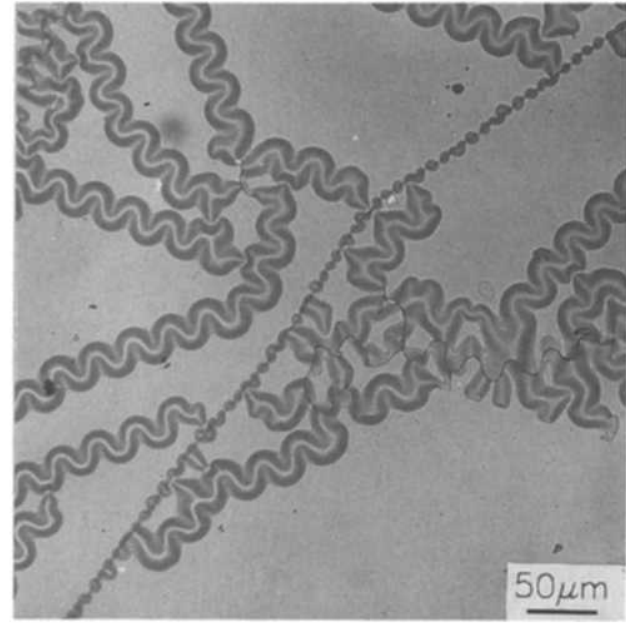

(b)

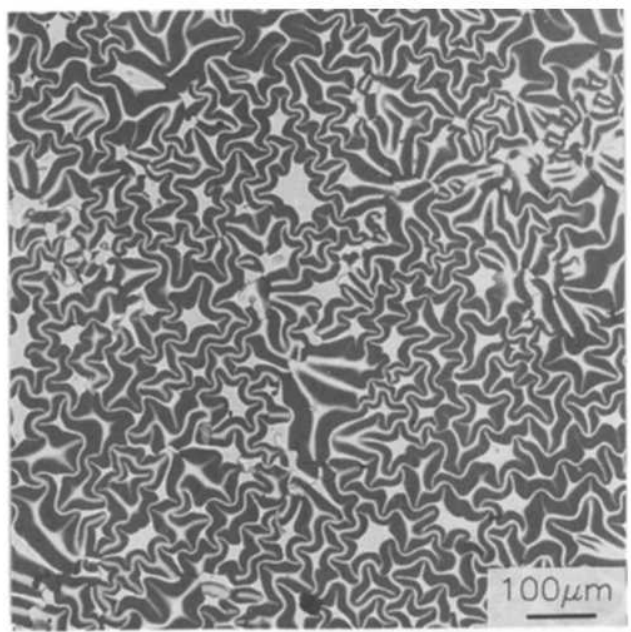

(d)

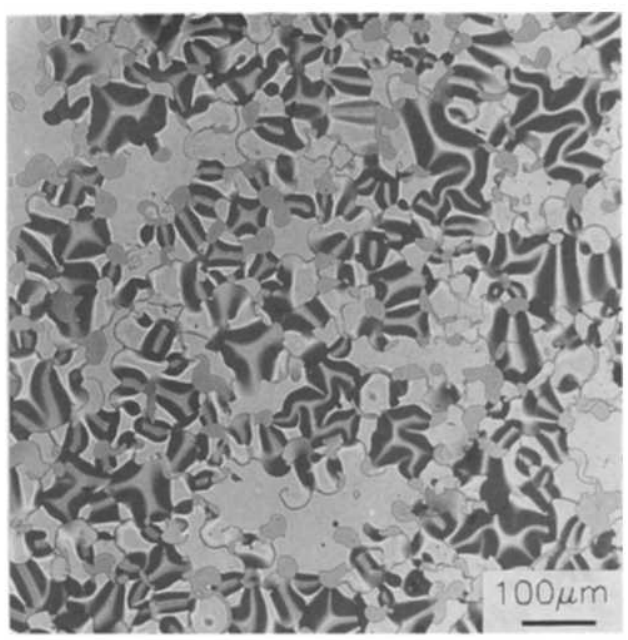

(f)

Fig. 5. Microstructures of the stress relief morphology in films deposited at negative bias voltages of -100 and $-500 \mathrm{~V}$ : (a) $-100 \mathrm{~V}$ for $1 \mathrm{~h}$; (b) $-500 \mathrm{~V}$ for $38 \mathrm{~min}$; (c) $-100 \mathrm{~V}$ for $90 \mathrm{~min}$; (d) $-500 \mathrm{~V}$ for $52 \mathrm{~min}$; (e) $-100 \mathrm{~V}$ for $2 \mathrm{~h}$; (f) $-500 \mathrm{~V}$ for $1 \mathrm{~h}$. 
film thickness or the negative bias voltage. The results of wavelength measurements are compiled in Fig. 6. (Zero wavelength in Fig. 6 means that no delamination can be observed.) Beyond the critical film thickness at which the delamination starts, the wavelength increases as the film thickness and the negative bias voltage are increased. The critical film thickness for the buckling to start also increases with increasing negative bias voltage. The rate of increase in the wavelength with respect to the film thickness is linearly proportional to the negative bias voltage as shown in Fig. 7 .

The film structure and properties are dependent on the negative bias voltage which determines the ion energy on the substrate [11]. One example is the compressive stress of the film. Figure 8 shows the measured compressive stress for various values of the negative bias voltage. The compressive stress of the film increases with

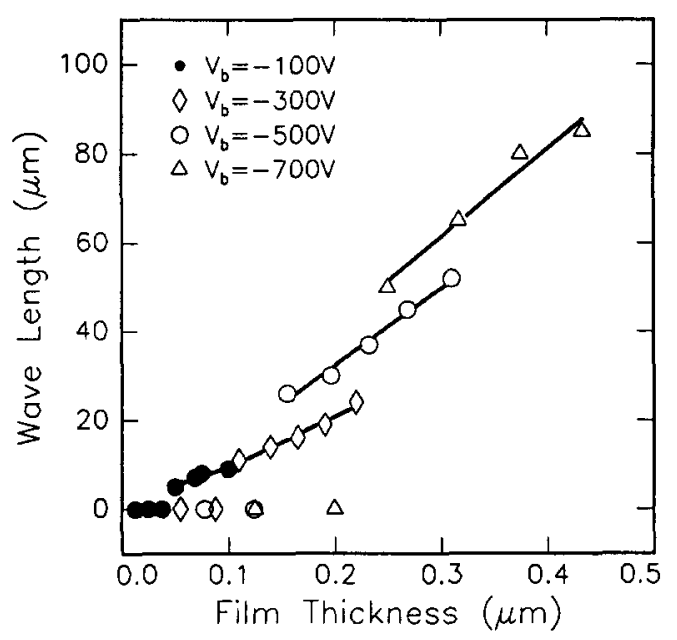

Fig. 6. Variation in the wavelength of sinusoidal buckling $v \mathrm{~s}$. the film thickness for various values of negative bias voltage.

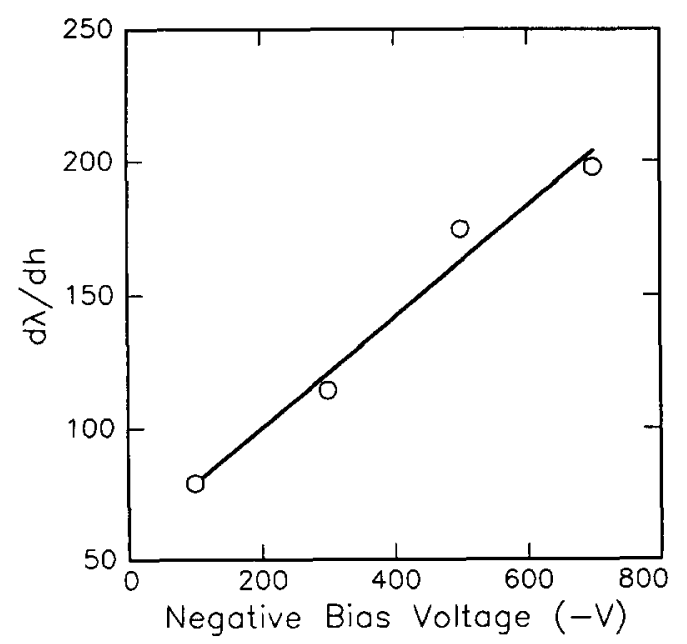

Fig. 7. Dependence of the rate of increase in wavelength with respect to the film thickness on the negative bias voltage. Here $\lambda$ is the wavelength and $h$ the film thickness.

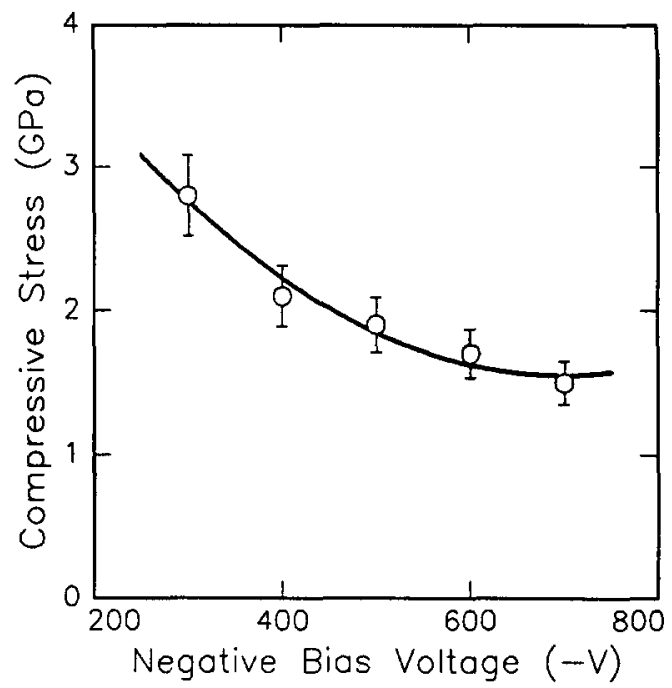

Fig. 8. Dependence of the internal stress on the negative bias voltage.

decreasing negative bias voltage. However, the adhesion of the film on the substrate also depends on the ion energy, because the ion energy determines the degree of intermixing at the interface between the film and the substrate [16]. Therefore both the film adhesion and the film properties should be taken into account to explain the dependence of the delamination behaviour on the negative bias voltage. The variation in the critical film thickness with negative bias voltage and the difference in cracking behaviour of thick films as shown in Fig. 5 should be explained in terms of both the variation in the film adhesion and the elastic energy due to the internal stress of the film.

As in previous work [9], it was observed that on blowing humid air onto the specimen, the sinusoidal buckling occurs at a higher propagation rate than in an ordinary atmosphere. However, it was impossible to measure the propagation rate quantitatively, because the sinusoidal buckling occurs rapidly. However, the wavelength was observed to be constant regardless of the propagation rate. Even though the delamination mechanism is not fully investigated in the present work, this observation suggests that the humidity affects only the film adhesion but not the wavelength of the sinusoidal buckling, because the adhesion should be significant to the propagation rate. It can be further assumed from this observation that the effect of the change in adhesion is not significant to the wavelength. If the wavelength is dependent on the film adhesion, the humidity that changes the film adhesion would also affect the wavelength. With this assumption, the wavelength measurement with respect to the negative bias voltage was used to identify the mechanical properties of the film.

Nir [9] has analysed the sinusoidal buckling behaviour using the linear theory of plates. Even though the analysis cannot offer an explanation of the origin of the 
sinusoidal buckling. Nir's phenomenological equation, eqn. (11) of ref. 9, can give us a relation between the wavelength, amplitude, internal stress and elastic modulus of the film. The parameters $k$ and $q$ in eqn. (11) of ref. 9 are respectively related to the amplitude of sinusoidal buckling, $A$, and the wavelength $\lambda$ as follows:

$A=\frac{\pi}{q}, \quad \lambda=\frac{2 \pi}{k}$

Hence, by inserting eqn. (2) into eqn. (7) of ref. 9 and rearranging it, one can obtain

$\frac{1}{4 A^{2}}+\frac{1}{\lambda^{2}}=\frac{1-v^{2}}{E} \frac{3 \sigma}{\pi^{2} h^{2}}$

where $E$ is the elastic modulus of the film, $v$ is the Poisson ratio, $\sigma$ is the internal stress and $h$ is the film thickness. Since the amplitude is about one-quarter of the wavelength regardless of the deposition condition,

$i^{2}=\frac{E}{1-v^{2}} \frac{5 \pi^{2} h^{2}}{3 \sigma}$

can be obtained. This equation shows that the wavelength of sinusoidal buckling is proportional to the film thickness and is inversely proportional to the square root of the stress. As can be seen in Fig. 8, the internal stress is inversely proportional to the negative bias voltage. The observed buckling behaviours with respect to the negative bias voltage and the film thickness are hence qualitatively consistent with this equation.

For films thicker than $0.1 \mu \mathrm{m}$ the stress of the film is generally independent of the film thickness [12]. Hence the rate of increase in the wavelength with respect to the film thickness, $\mathrm{d} \lambda / \mathrm{d} h$, is proportional to the square root of $E /\left(1-v^{2}\right) \sigma$. Using the data of Figs. 7 and 8, the elastic constant $E /\left(1-v^{2}\right)$ was estimated to increase with increasing negative bias voltage from $2232 \mathrm{GPa}$ at $-300 \mathrm{~V}$ to $3571 \mathrm{GPa}$ at $-700 \mathrm{~V}$. Because the Poisson ratio is positive, these values are the lower limits of the elastic modulus $E$. The elastic moduli of metals, carbides and nitrides are of the order of $10^{2} \mathrm{GPa}[17,18]$. The elastic modulus of diamond is about $900 \mathrm{GPa}$ [17]. The estimated elastic modulus of DLC films is almost one order of magnitude higher than those of other materials and two to four times larger than that of diamond.

Even though elastic modulus data of DLC films have not yet been reported, these calculated values are thought to be overestimations as deduced from the values of other ceramic materials. The errors in the measurement of the stress, the wavelength and the film thickness cannot explain this large disagreement. One possibility is that the previous theory was based on the thin plate having free surfaces on both sides, while the DLC film is deposited on the substrate. Hence a part of the elastic energy should be dissipated during the separation of the film from the substrate. The previous theory did not consider this elastic energy dissipation. A more elaborate theory is thus needed for the quantitative analysis.

\section{Conclusions}

The most significant result shown in this paper is that the stress relief behaviour is sensitive to the film properties as well as the film thickness. The wavelength of sinusoidal buckling of DLC films on glass substrates was found to be dependent on process parameters such as the negative bias voltage in r.f.-PECVD. The simple elastic theory of a thin plate under isotropic compressive stress [9] was applied to describe the behaviour in terms of the film thickness and film properties. The buckling behaviour can be well described by this phenomenological expression in a qualitative manner.

The possibility of measuring the mechanical properties of DLC films using their stress relief behaviour was suggested in this paper. Using the phenomenological equation derived by Nir [9], the elastic modulus of DLC films was estimated to be of the order of $10^{3} \mathrm{GPa}$. However, this value is unlikely to be reasonable, because the elastic moduli of other ceramic materials, including diamond, are one order of magnitude lower. Since the previous theory did not include the effect of adhesion between the film and the substrate, the quantitative analysis needs a more elaborate theory on the sinusoidal buckling of the film.

\section{Acknowledgments}

The authors are grateful to Jae-Won Ryu for measurement of the internal stress and to Seong-Ryong Cho for careful reading of the manuscript. This work was financially supported by the Korea Ministry of Science and Technology and the IIJin Diamond Company.

\section{References}

1 S. Aisenberg and R. Chabot, J. Appl. Phys., 42 (1971) 2953.

2 S. Miyake and R. Kaneko, Thin Solid Films, 212 (1992) 256.

3 A. Bubenzer, B. Dischler and A. Nyaiesh. Thin Solid Films, 91 (1982) 81

4 H. C. Tsai and D. B. Bogy, J. Vac. Sci. Technol. A, 5 (1987) 3287.

5 P. I. Perov, V. I. Polyakov, O. N. Ermakova, M. G. Ermakov, V. M. Elinson and V. V. Sleptosov, Proc. Ist Int. Conf. on the Applications of Diamond Films and Related Materials, Auburn. August 1991, Elsevier, Amsterdam, 1991, p. 761.

6 J. C. Augus, P. Koidl and S. Domitz, in J. Mort and F. Jansen (eds.), Plasma Deposited Thin Films, CRC Press, Boca Raton, FL, 1986, p. 89.

7 C. Weissmantel, C. Schürer, F. Fröhlich, P. Grau and H. Lehmann, Thin Solid Films, 61 (1979) L5. 
8 C. Weissmantel, G. Reisse, H.-J. Erler, F. Henny, K. Bewilogua, U. Ebersbach and C. Schürer, Thin Solid Films, 63 (1979) 315.

9 D. Nir, Thin Solid Films, 112 (1984) 41.

10 M. David, R. Padiyath and S. V. Babu, AIChE J., 37 (1991) 367.

11 A. Bubenzer, B. Dischler, G. Brandt and P. Koidl, J. Appl. Phys., 54 (1983) 4590.

12 K. L. Chopra, Thin Film Phenomena, McGraw-Hill, New York, 1969, p. 311.

13 J. W. Hutchinson, M. D. Thouless and E. G. Liniger, Acta Metall. Mater., 40 (1992) 295.
14 A. Brenner and S. Senderoff, J. Res. NBS, 42 (1949) 105.

15 D. A. Skoog and J. J. Leary, Principles of Instrumental Analysis, Saunders College Publishing, Fort Worth, TX, 4th edn., 1992, p. 695.

16 C. Weissmantel, K. Breuer and B. Winde, Thin Solid Films, 100 (1983) 383.

17 R. Kieffer and F. Benesovsky, in M. Grayson (ed.), Kirk-Othmer Encyclopedia of Chemical Technology, Vol. 4, Wiley, New York, 1978, p. 480.

18 E. A. Brandes (ed.), Smithells Metals Reference Book, Butterworths, London, 6th edn., 1983, p. 15-2. 